\title{
INFORMACIÓN PALEOBIOLÓGICA Y PALEOAMBIENTAL INFERIDA A PARTIR DE LAS ICNITAS DE DINOSAURIOS: PROBLEMAS, LÍMITES Y PERSPECTIVAS
}

\author{
José Joaquín MORATALLA', José Luis SANZ' y \\ Santiago JIMÉNEZ
}

\author{
' Unidad de Paleontología. Dto. Biología. Fac. Ciencias. Universidad Autónoma. \\ Cantoblanco. 28049 Madrid. \\ 2 Iberdrola. Sección de Mineralogía y Paleontología. Gardoquí. 8. 48008 Bilbao.
}

Moratalla, J. J., Sanz, J. L. y Jiménez, S. 1997. Información paleobiológica y paleoambiental inferida a partir de las icnitas de dinosaurios: problemas, límites y perspectivas. [Paleobiological and paleoenvironmental information inferred from dinosaur tracks: problems, limits and perspectives]. Revista Española de Paleontología, 12 (2), 185-196. ISSN 0213-6937.

\begin{abstract}
The morphology of a dinosaur track (and, in general, of a tetrapod one) is strongly influenced by four factors: autopodial anatomy, substrate, locomotion and preservation. One of the main objectives of Paleoichnology is the evaluation of interaction among these factors, which should allow the identification of the trackmaker. These factors determine the nature and limits of the inferences to be made of track information. Any study on footprint identification needs an evaluation of the variability, which may be quite major, as several examples in the fossil record show.

The information to be inferred from dinosaur tracks is of two kinds: paleobiological and paleoenvironmental. The first kind derives from the study of 1) isolated tracks that yield information about foot anatomy; 2) the trackway, yielding information about locomotion and 3) association of trackways, yielding information about possible group behavior and ecology. Analyses of track morphology have normally been carried out on footprint outlines. However, outlines do not give a threedimensional picture of the footprint. Trackway of theropod and ornithopod dinosaurs, despite their different pelvic construction, show a similar footprint rotation pattern (inward), different to that of the sauropods (outward). The transition from a bipedal stance to an optional quadrupedal gait in ornithopods implies a low energy expenditure for these dinosaurs.

The paleoenvironmental information highlights the conditions necessary for the formation/preservation of the tracks. For this it is necessary to consider the sedimentary context and the peculiarities of ichnological preservation (undertrack phenomena). The tracksites, and the megatracksites in particular, allow the evaluation of the ichnofaunal composition from both temporal and spatial perspectives. However, such an ichnofaunistic census has preservational biases influenced by two factors: animal activity and size.
\end{abstract}

Keywords: Paleoichnology, dinosaur tracks, Paleobiology, Paleoecology.

\section{RESUMEN}

La morfología de una icnita de dinosaurio (y, en general, la de un tetrápodo) está fuertemente influida por cuatro factores: anatomía autopodial, sustrato, locomoción y conservación. Uno de los principales objetivos de la Paleoicnología debe ser el análisis de la interacción entre estos factores, lo que permitiría, en la medida de lo posible, la identificación del autor. Estos factores determinan la naturaleza y límites de la información icnológica inferida. Así, cualquier estudio de identificación icnológica necesita una evaluación de la variabilidad. Existen numerosos ejemplos en el registro fósil que muestran una alta variabilidad incluso dentro de un mismo rastro, por lo que la evaluación de la misma debe ser anterior a cualquier inferencia sobre variabilidad intra o interespecífica que se lleve a cabo.

La información inferida a partir de las icnitas de dinosaurios se divide en dos grandes apartados: 1) información paleobiológica y 2) información paleoecológica. La primera categoría procede del estudio de 1) icnitas aisladas, que proporcionan información sobre anatomía autopodial, 2) rastros, que aportan información sobre locomoción y 3) asociación de rastros, que proporcionan información sobre posible comportamiento en grupo y ecología. Los análisis de morfología icnológica se han llevado normalmente a cabo sobre contornos de icnitas. No obstante, éstos no proporcionan un buen marco tridimensional de la huella. 
Los rastros de dinosaurios terópodos y ornitópodos, a pesar de su diferente construcción pélvica, muestran un patrón similar de rotación de las icnitas (interna), a diferencia de los saurópodos (externa). La transición de una locomoción bípeda a una cuadrúpeda en ornitópodos lleva consigo un bajo coste energético para estos dinosaurios.

La información paleoambiental obtenida a partir de las icnitas se centra fundamentalmente en las condiciones necesarias para la formación/conservación de las mismas. Es necesario considerar el contexto y las singularidades de la conservación icnológica (fenómeno de laa subimpresiones). Los yacimientos icnológicos y, en especial, los megayacimientos permiten evaluaciones icnofaunísticas en una perspectiva espacial y temporal. Sin embargo, estas estimaciones llevan consigo unos sesgos conservacionales influidos principalmente por dos factores: la tasa de actividad animal y el tamaño.

Palabras clave: Paleoicnología, icnitas de dinosaurios, Paleobiología, Paleoecología.

\section{INTRODUCCIÓN}

La probable diferencia entre un icnólogo experto y un principiante es que éste último tiende a interpretar icnitas diferentes como producto de distintos tipos de animales, mientras que el primero intentará reconocer, o al menos interpretar hasta cierto grado, las variaciones en la morfología icnológica que dependen de diferentes factores de sesgo: condiciones del sustrato, comportamiento del animal, conservación, etc. Así, un icnólogo experto puede llegar a distinguir entre los diferentes tipos de huellas que es capaz de producir un animal actual y también a grandes rasgos el tiempo transcurrido desde su formación. Un buen icnólogo necesita mucha experiencia, capacidad de observación y de deducción. Por consiguiente, uno de los objetivos más importantes de un paleoicnólogo tendría que ser la aplicación al pasado del conocimiento de la ciencia icnológica en restos actuales, poniendo de manifiesto aquellos factores de sesgo que han podido alterar la morfología icnológica original. Estos criterios han estado siempre subyacentes en numerosos autores (Peabody, 1955; Baird, 1957; Sarjeant, 1975, 1989; Haubold, 1984; Lockley, 1986; Leonardi, 1987), que han abordado la problemática de la Paleoicnología.

El número de hallazgos de nuevos yacimientos de icnitas ha crecido enormemente en los útimos años. En consecuencia, la paleoicnología de dinosaurios es una rama que se ha convertido progresivamente en un complemento muy importante para entender la biología de los dinosaurios, y despierta cada vez más el interés de los

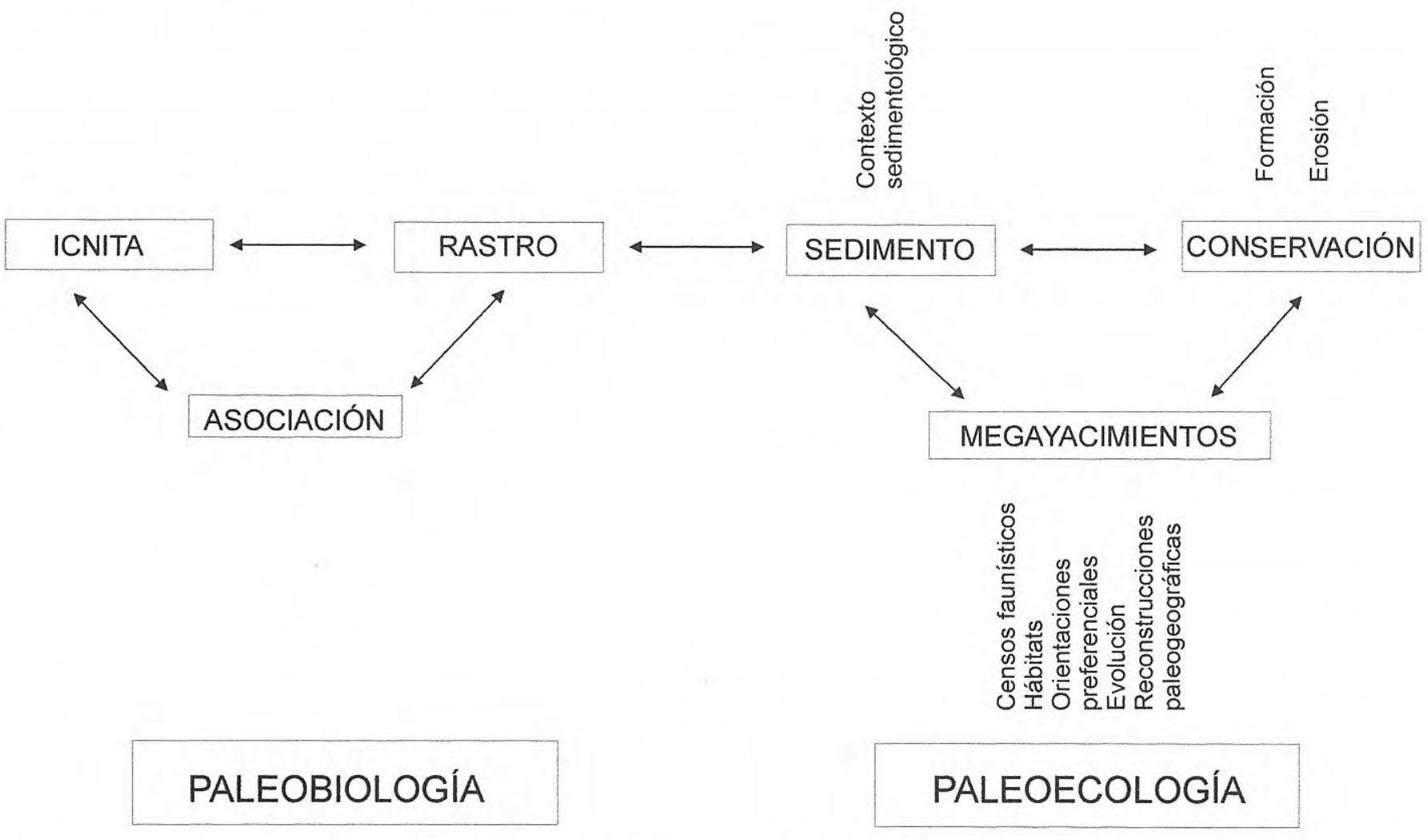

Figura 1. Esquema de los dos tipos principales de información (Paleobiología y Paleoecología) que podemos extraer del estudio de las icnitas de vertebrados (véase texto para más detalles). 


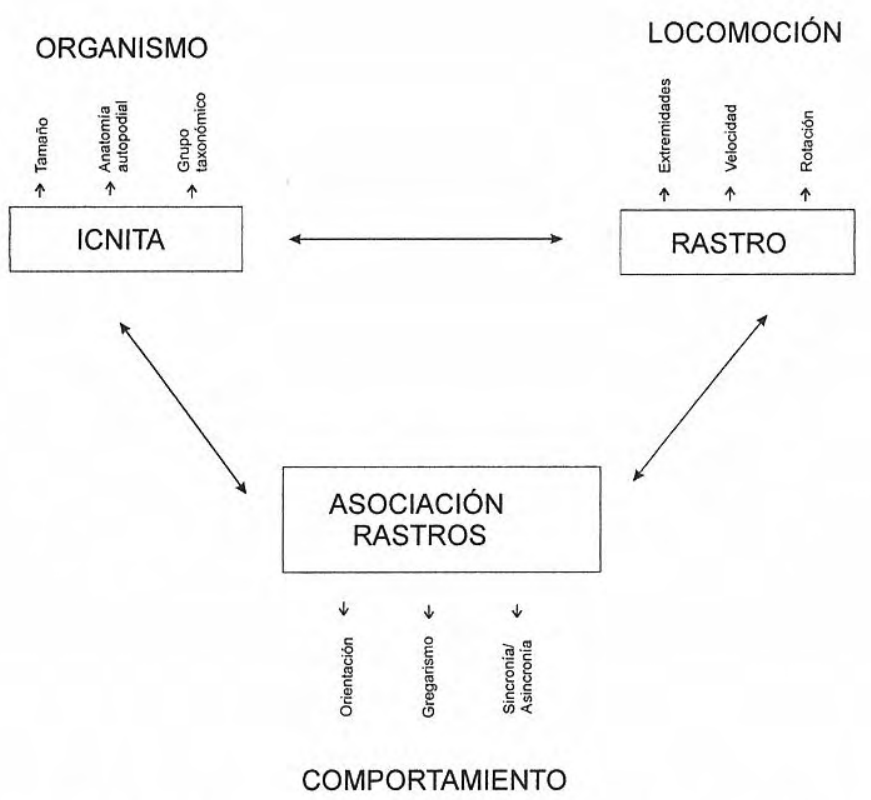

Figura 2. Esquema de los tres grandes tipos de información paleobiológica proporcionada por las icnitas de vertebrados (véase texto para más detalles).

paleontólogos en particular, y del público en general, interés que se hace extensivo a la propia información generada por su estudio. Esta información puede ser agrupada en dos grandes apartados: Paleobiología y Paleoecología (Fig. 1). Pero la naturaleza de los documentos (restos indirectos) obliga a considerar cuidadosamente los límites y problemas que conllevan. Parece claro que las inferencias biológicas realizadas a partir de material directo (por ejemplo, un hueso) están principalmente relacionadas con su morfología (teniendo en cuenta también los casos de deformaciones u otros cambios fosildiagenéticos). Por el contrario, las icnitas están producidas por la interacción en vida del organismo con el sustrato, y su morfología estará fuertemente influida por la naturaleza de esta interacción. Además, existirán azares conservacionales que son inherentes a la naturaleza icnológica (por ejemplo, el fenómeno de las subimpresiones) que no está presente en el registro fósil directo.

El objetivo principal de este trabajo, tomando como base las icnitas de dinosaurios, es poner de manifiesto cuál es el tipo de información que puede ser potencialmente extraída a partir de las icnitas fósiles y cuáles son los límites y problemática concreta que genera su estudio.

\section{INFORMACIÓN PALEOBIOLÓGICA}

La Fig. 2 muestra esquemáticamente el tipo de información paleobiológica que se puede extraer a partir del estudio paleoicnológico de dinosaurios. En primer lugar, está la consideración de una icnita de forma individualizada. En segundo lugar, el estudio de un rastro y, por último, la asociación de diversos rastros.

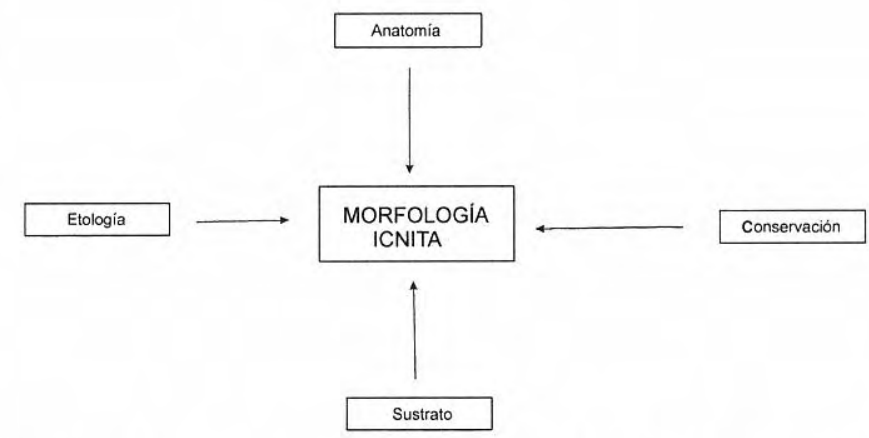

Figura 3. La morfología de una icnita depende fundamentalmente de los cuatro factores que se encuentran en este esquema. Entender el nivel de interactuación de los mismos es un problema de difícil resolución, aunque esencial en la consideración de la variabilidad icnológica (véase texto para más detalles).

\section{Icnita individual}

Una icnita individual aporta información sobre la anatomía autopodial y, por consiguiente, sobre el autor de la misma, aunque la identificación del dinosaurio responsable está también basada en ciertos caracteres del rastro. Este proceso de identificación plantea numerosos problemas ya que la morfología icnológica no es consecuencia solamente de la del autópodo responsable, sino también de factores extraorgánicos de difícil evaluación: velocidad de progresión, equilibrio, condiciones del sustrato (grado de compactación, humedad), azares de conservación, etc. Así, la morfología de una icnita depende de 4 factores principales: anatomía, sustrato, etología y conservación (Fig. 3).

\section{Anatomía}

La morfología autopodial varía según las especies. Sin embargo, el pie es una estructura orgánica fuertemente adaptada a la locomoción, lo que sugiere que diferentes especies de dinosaurios pueden haber presentado una construcción autopodial relativamente similar, haciendo difícil la distinción morfológica de sus respectivas icnitas. Por ejemplo, las icnitas de terópodos están típicamente formadas por impresiones digitales esbeltas y de terminación distal acuminada. Sin embargo, ornitópodos de pequeño tamaño poseen falanges ungueales mucho más acuminadas que, por ejemplo, un gran ornitópodo de estructura autopodial graviportal, como es Iguanodon bernissartensis. Por consiguiente, si aplicamos simplemente criterios de morfología digital, estas pequeñas huellas de ornitópodos podrían ser malinterpretadas y atribuidas a terópodos. Existen además otras variaciones morfológicas causadas por factores estrictamente anatómicos: 1) cambios de forma durante la ontogenia y 2) patologías. Las primeras son muy difíciles de comprobar en el registro fósil aunque se conocen yacimientos donde existe un amplio rango de tamaños para un mismo morfotipo icnológico (Moratalla, 1993; Lockley 1994; Lockley y Hunt, 1995). En cuanto a las segundas, se han descrito al menos dos tipos de patologías: mutilación/deformación (Abel, 1935; Ishigaki, 


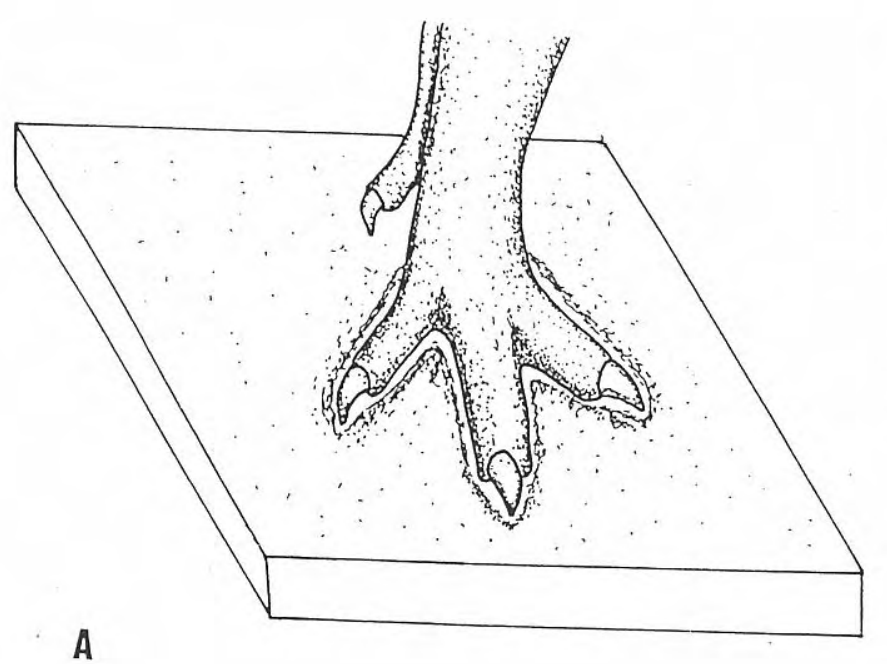

B

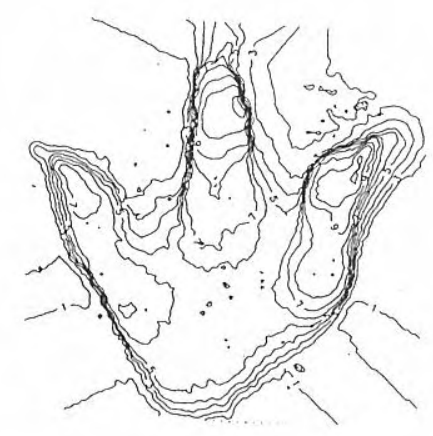

C

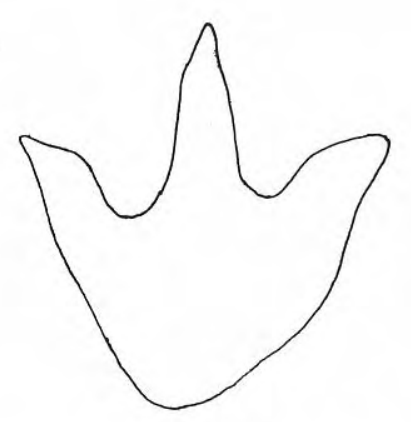

Figura 4. Esquema de la transferencia de información desde el autópodo (A), icnita tridimensional (B, según Farlow, 1993), hasta el contorno lineal de la icnita ( $\mathbf{C}$, el objeto tradicional de los análisis morfométricos). Probablemente, en un futuro cercano dispongamos de potentes herramientas de análisis topográfico de la superficie total de las pisadas que permitan una aproximación morfológica mucho más completa. Sin embargo, hasta el momento, cualquier análisis de este tipo no ha aportado nueva información.

1986) y alternancia de pasos cortos y largos (cojera) (Lockley et al., 1994a).

Aunque a priori sencilla, la discriminación entre diferentes morfotipos de icnitas tridáctilas de dinosaurios es uno de los problemas más intrincados de la Paleoicnología. Este proceso se ha llevado a cabo fundamentalmente en base a caracteres métricos y no métricos (Moratalla et al., 1988). A pesar de la amplia utilización de caracteres discretos (morfología digital, terminación distal de los dedos, forma del talón, etc.,), las propuestas más significativas en este sentido han tendido a la cuantificación de resultados y se han basado en caracteres métricos (variables e índices), con los que se han empleado diversos tipos de análisis multivariantes (Moratalla et al., 1988; Demathieu y Wright, 1988; Weems, 1992). Estas variables e índices están basados en el contorno de la icnita, y en pocos casos han proporcionado resultados satisfactorios, con dudosos valores umbrales discriminatorios incluso entre icnitas de terópodos y de ornitópodos (una revisión de estos métodos puede verse en Moratalla, 1993). Las causas de estos resultados en los análisis discriminantes son diversas y complejas. El origen del problema puede centrarse probablemente en el concepto de morfología icnológica. Una icnita constituye por sí misma una estructura tridimensional cuyo análisis topográfico puede llegar a ser realmente complejo y sofisticado. Hersberger y Farlow (1990) han llevado a cabo esfuerzos interesantes en este sentido. Sin embargo, la transferencia de información anatómica desde el autópodo hasta el contorno icnológico (el objeto de los análisis) lleva consigo una pérdida de información biológica, en la que podemos considerar dos tipos de transferencias de información (Fig. 4):

1) La primera nos lleva desde la morfología autopodial (cuya forma en realidad nos interesa discernir) hasta la icnita tridimensional. Esta primera transformación está influida por aquellos factores de variabilidad mencionados anteriormente: locomoción, sustrato y conservación.

2) La segunda nos lleva a una idealización de la compleja estructura tridimensional en una silueta lineal. Este paso, aparentemente simple, está dificultado ocasionalmente por factores de conservación que impiden observar nítidamente el contorno, a veces difuso. A su vez, está generado por la interpretación del propio paleoicnólogo, y lleva consigo un pequeño porcentaje de subjetividad (Thulborn, 1990; p. 92).

Sin duda, el incremento de la informática como herramienta de trabajo, llevará en el futuro a una mayor capacidad de cuantificación-comparación morfológica, con la que tal vez se resuelvan algunos problemas actuales.

Una icnita aislada proporciona también información sobre el tamaño del autor. De hecho, el tamaño de la huella se correlaciona con el tamaño del pie, y éste con el tamaño corporal. Sin embargo, esta correlación aparentemente tan simple presenta diversos problemas debido a que la relación tamaño de la icnita con el tamaño del cuerpo depende principalmente de 1) grupo taxonómoco del autor, 2) apoyo del pie en el sustrato y 3) variabilidad ontogénica. Se han propuesto diversos métodos de estimación de la altura de la extremidad (h). El primero fue propuesto por Alexander (1976), quien estimó h como aproximadamente 4 veces la longitud de la huella. Posteriormente, Sanz et al. (1985) propusieron un análisis estadístico alternativo basado en restos esqueléticos de 
ornitópodos y en el que $\mathrm{h}=3,91 \mathrm{FL}+10,94$, donde FL es la longitud de la icnita. Este método proporciona una altura de la extremidad ligeramente mayor que la obtenida a partir del método de Alexander. En cualquier caso, esta altura depende fundamentalmente del taxon dinosauriano ya que existen diversos tipos de adaptación: formas corredoras, más o menos graviportales, etc. Aquí habría que tener en cuenta la variabilidad ontogénica, responsable tal vez en algún caso de una diferente proporción.

Estos factores llevaron a Thulborn y Wade (1984) y Thulborn (1990) a proponer una serie de ecuaciones alométricas para los distintos tipos de dinosaurios:

Pequeños terópodos $(\mathrm{FL}<25 \mathrm{~cm}): \mathrm{h}=4,5 \mathrm{FL}$

Grandes terópodos (FL $>25 \mathrm{~cm}): \mathrm{h}=4,9 \mathrm{FL}$

Pequeños ornitópodos (FL $<25 \mathrm{~cm}): \mathrm{h}=4,8 \mathrm{FL}$

Grandes ornitópodos (FL $>25 \mathrm{~cm}$ ): $\mathrm{h}=5,9 \mathrm{FL}$ $4,6 \mathrm{FL}$

Pequeños dinosaurios en general $(\mathrm{FL}<25 \mathrm{~cm})$ : $\mathrm{h}=$ $5,7 \mathrm{FL}$

Grandes dinosaurios en general $(\mathrm{FL}>25 \mathrm{~cm}): \mathrm{h}=$

El método de Thulborn parece ser conceptualmente el más correcto debido a que evita en cierto modo el problema de la generalización. Sin embargo, presenta también diversos problemas. En efecto, una incorrecta identificación icnológica lleva consigo una incorrecta estimación de $\mathrm{h} y$, por otra parte, el valor umbral para elegir uno u otro grupo taxonómico está basado en el tamaño de la icnita. Debemos esperar, por consiguiente, ulteriores análisis biométricos del esqueleto que permitan un ajuste mucho más preciso de este problema.

\section{Sustrato}

Las condiciones del sustrato forman un elemento de profunda significación morfológica ya que afectarán al modo en que éste registrará las improntas. El grado de humedad, cohesión y textura del sedimento es importante para el registro de caracteres morfológicos (Bromley, 1990: p. 139, fig. 8.1; Lockley y Hunt, 1995; Sarjeant y Leonardi, 1987). Las condiciones ideales están relacionadas con un elevado grado de plasticidad. Existen también características extrínsecas a la composición del sustrato, como, por ejemplo, posibles alteraciones locales, inclinación, obstáculos ocasionales, etc. La interacción de estos factores producirá una variabilidad realmente notable. La valoración de la misma no es un problema de fácil resolución. Aquí parece necesaria la observación y estudio de eventos actuales que permitan estimar su importancia relativa (Cohen et al., 1991). La ventaja, en este caso, es la posibilidad de experimentación con formas actuales en condiciones cambiantes y controladas de diferentes tipos de sustratos. La extrapolación de estas experiencias al registro fósil sería indudablemente una importante herramienta interpretativa en este aspecto.

\section{Comportamiento}

El comportamiento del animal es muy importante para la morfología de la huella que produce. Dollo (1883) mostró un ejemplo famoso de la morfología icnológica

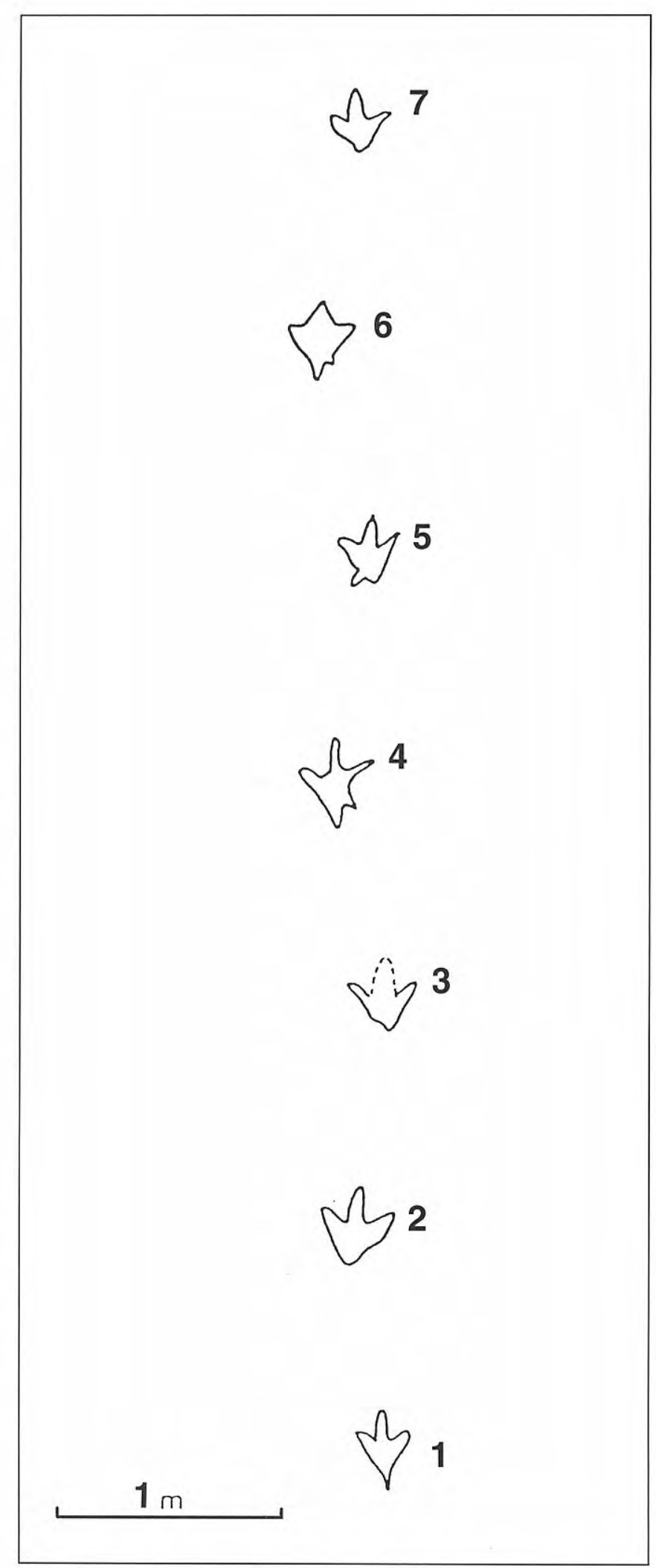

Figura 5. Esquema del rastro LCA-R34 del yacimiento de Los Cayos (Cornago, La Rioja). Obsérvese la variabilidad existente en las distintas impresiones producidas por el mismo animal. Esta pista es uno de los ejemplos más significativos de cómo la variabilidad intraindividual afecta cualquier tipo de análisis. 


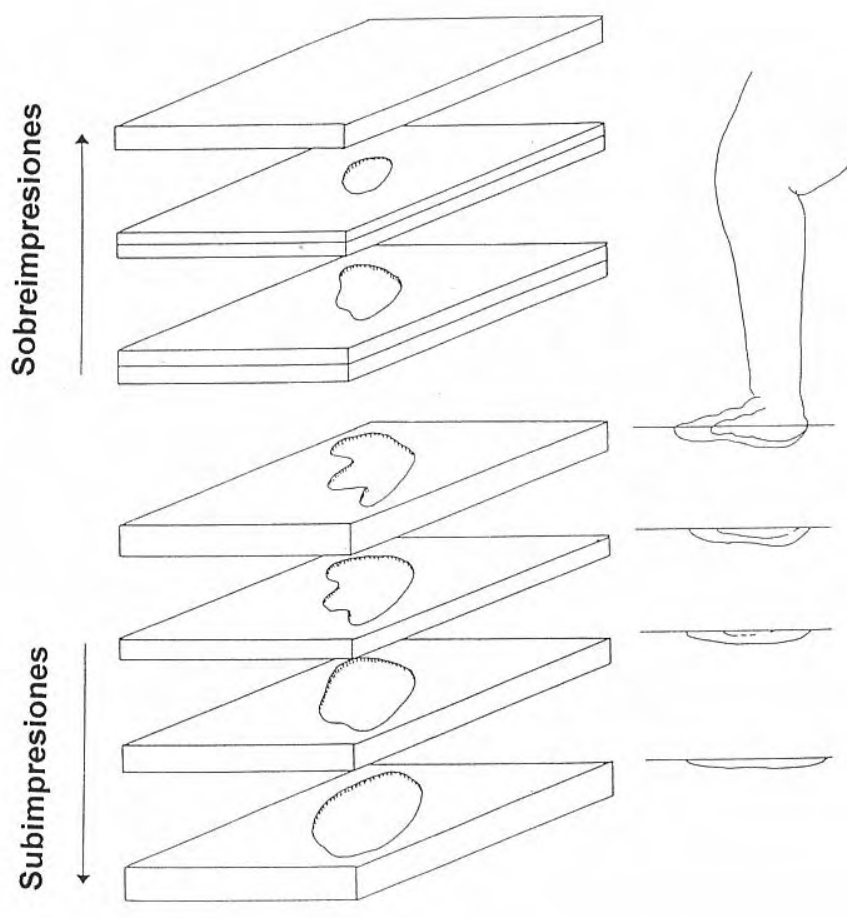

Figura 6. Cuando un animal pisa en el sustrato puede deformar al mismo tiempo diversas láminas sedimentarias. De este modo, una sola icnita real puede ser responsable de la presencia de diversas subimpresiones. La morfología de las mismas será más difusa cuanto más alejada esté de la icnita real. Además de estas subimpresiones ("undertracks"), la huella real puede ser rellenada por sedimento adicional formándose una réplica en relieve de la misma (según Langston, 1979).

dependiendo de que el animal estuviese parado, andando o corriendo. Además de la velocidad de progresión, es muy importante también el equilibrio, la realización de un giro y el modo de apoyo del autópodo sobre el sustrato.

Uno de los objetivos a resolver es el conocimiento de cómo la variabilidad intraespecífica e intraindividual afecta a la morfología de la icnita. El yacimiento de Los Cayos A (Cornago, La Rioja) contiene algunos ejemplos muy significativos (Moratalla, 1993). La Fig. 5 es un dibujo esquemático del rastro LCA-R34, perteneciente a un dinosaurio terópodo. Este rastro, constituido por 7 icnitas, es uno de los más interesantes de toda la Cuenca de Cameros ya que es un buen ejemplo de variabilidad intraindividual. La primera icnita (LCA-R34/1) es tridáctila, con un talón muy elongado proximalmente, causado por el apoyo de la zona distal del metatarso. Las dos siguientes icnitas (LCA-R34/2 y LCA-R34/3) son también tridáctilas, con un contorno proximal menos elongado y con una ligera escotadura medial en el área talonar. Por el contrario, LCA-R34/4, LCA-R34/5 y LCAR34/6 son tetradáctilas, ya que en todos los casos existe una impresión medial producida por el hallux. Mientras LCA-R34/4 presenta unos dígitos relativamente elongados y muy delgados, con una impresión posterior muy elongada del talón, LCA-R34/5 presenta, por el contrario, unos dígitos más robustos y una menor elongación proximal. La morfología de los dedos de LCA-R34/6 es igualmente muy robusta, más incluso que en la anterior icnita, y el talón vuelve a presentar una nítida y prolongada impresión proximal. Por último, LCA-R34/7, última icnita de esta pista, presenta de nuevo una morfología netamente tridáctila, con un contorno proximal relativamente redondeado, aunque conservando nítidamente la impresión de una escotadura medial. Las diferentes morfologías de este rastro, debidas probablemente a un modo de apoyo diferencial durante la marcha y/o a diferencias locales en las condiciones del sustrato, no parecen consecuencia de cambios en las condiciones cinéticas de la locomoción, ya que las medidas obtenidas in situ para pasos y zancadas proporcionan valores relativamente constantes a lo largo de la misma. Igual ocurre con la dirección de movimiento, ya que el patrón de la pista es recto. Este rastro constituye un buen ejemplo de la necesidad de aplicar una metodología muy rigurosa al estudio de las huellas del pasado. En caso contrario, podría ocurrir que si encontrásemos, por ejemplo, las icnitas LCA-R34/2 y LCA-R34/5 en diferentes afloramientos las interpretáramos como producidas por dos tipos distintos de dinosaurios. Sin embargo, aquí tenemos la evidencia de cómo el mismo organismo puede producir un elevado rango de variabilidad morfológica. Existen otros casos relativamente similares en la Cuenca de Cameros. Esta variabilidad intraindividual puede ser muy elevada, por encima del $80 \%$ incluso en rastros muy bien preservados y aparentemente homogéneos (Moratalla, 1993).

\section{Conservación}

Otro importante problema de la icnología se refiere a factores inherentes a la conservación. La formación de subimpresiones ("undertracks") fue citada por primera vez por Hitchcock (1858). Cuando un autópodo pisa en el sedimento produce una deformación en el mismo que se hace extensiva también a las láminas sedimentarias subyacentes. Este fenómeno da lugar a una columna de deformación, que constituye en realidad la estructura de carga provocada por la pisada (Fig. 6). De este modo, un mismo evento dinámico puede producir diversas impresiones apiladas unas sobre otras con un origen biogénico común. Solamente el nivel sedimentario donde el animal pisa constituye la icnita real, donde, si las condiciones originales son lo suficientemente adecuadas, se podrán apreciar estructuras anatómicas tales como impresiones tegumentarias, almohadillas, etc. La morfología de las deformaciones subyacentes a la icnita real (subimpresiones, calcos o "undertracks") dependerá de la presión ejercida, de las características de cada lámina y de la distancia al nivel de la pisada verdadera. En otras palabras, la difuminación de la morfología de la huella será directamente proporcional a la distancia sedimentaria a la impresión original. En efecto, cuanto más nos alejemos de la misma más difuminada estará la impresión (Fig. 6): los bordes serán menos netos, la pendiente cerca del contorno tenderá a ser más suave y el tamaño general será algo mayor. La existencia de subimpresiones nos lleva a 


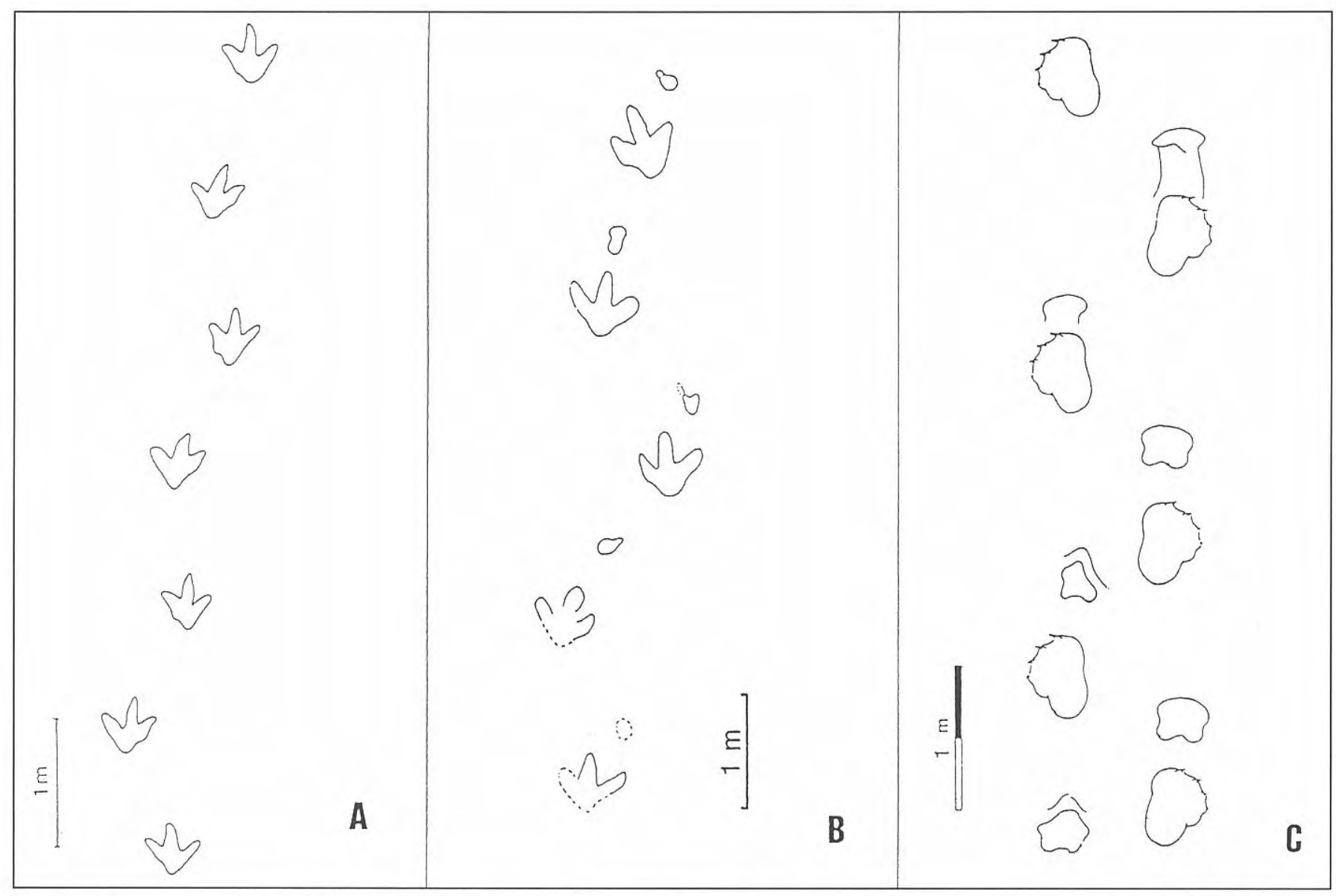

Figura 7. Tres ejemplos de rastros de dinosaurios. A, terópodo (Cornago, La Rioja); B, ornitópodo (Regumiel de la Sierra, Burgos); C, rastro de saurópodo identificado como la icnoespecie Brontopodus birdi Farlow et al. (1989). Obsérvese cómo las icnitas del terópodo y ornitópodo están orientadas hacia el interior de la línea media del rastro (andar zambo) y que, por el contrario, en el caso del saurópodo están orientadas hacia fuera (andar estevado).

consideraciones interesantes: 1) la subimpresión queda inmediatamente fuera del alcance de los agentes destructivos más inmediatos, lo que aumenta la posibilidad de conservación en el registro fósil; 2) un animal puede pisar un sedimento cuando las condiciones para la conservación de sus huellas no son adecuadas (imaginemos, por ejemplo, un sustrato muy húmedo), y a pesar de que las huellas originales sean borradas, las subimpresiones que éstas han generado pueden conservarse; 3) si esto es así, el número esperable de subimpresiones en el registro fósil es teóricamente superior al de las huellas verdaderas, por dos razones: a) el número de subhuellas es teóricamente mayor (una única pisada real puede formar varias subimpresiones) y b) como ya expusimos, presentan una mayor potencialidad de conservación en el registro fósil. Además de las icnitas verdaderas y las "undertracks" pueden existir impresiones de relleno debido al aporte de sedimentos que cubran el hueco producido por la pisada original. La réplica de la icnita original suele conservar muy bien los caracteres icnológicos y, en ocasiones, constituyen buenos y conspicuos ejemplos de icnitas de dinosaurios. El fenómeno "undertrack" en general no ha merecido excesiva atención por parte de los paleoicnólogos, aunque actualmente se está profundizando en su entendimiento (Lockley, 1991; Lockley y Hunt, 1995)). Esto ha permitido reinterpretar algunos yacimientos famosos, tal como el del saurópodo nadador de Bird (1944), como un caso explicable aplicando el concepto de las subimpresiones (Lockley y Rice, 1990), aunque la posibilidad de un saurópodo nadador podría seguir abierta en otros casos (Ishigaki, 1989).

En consecuencia, un mismo animal es capaz potencialmente de producir diferentes tipos de huellas dependiendo de su comportamiento o de las condiciones del sustrato y, al contrario, un mismo tipo de icnita puede ser producida por formas diferentes dependiendo del papel interactuante de estos factores. Éstos obligan a que la identificación no pueda llevarse a cabo a nivel específico, sino solamente a nivel de grandes taxones (orden, famila, etc.).

\section{Rastro}

El estudio de un rastro proporciona información fundamentalmente sobre locomoción. La mayoría de los rastros de dinosaurios bípedos (tanto terópodos como ornitópodos) son relativamente estrechos (ángulo de paso 


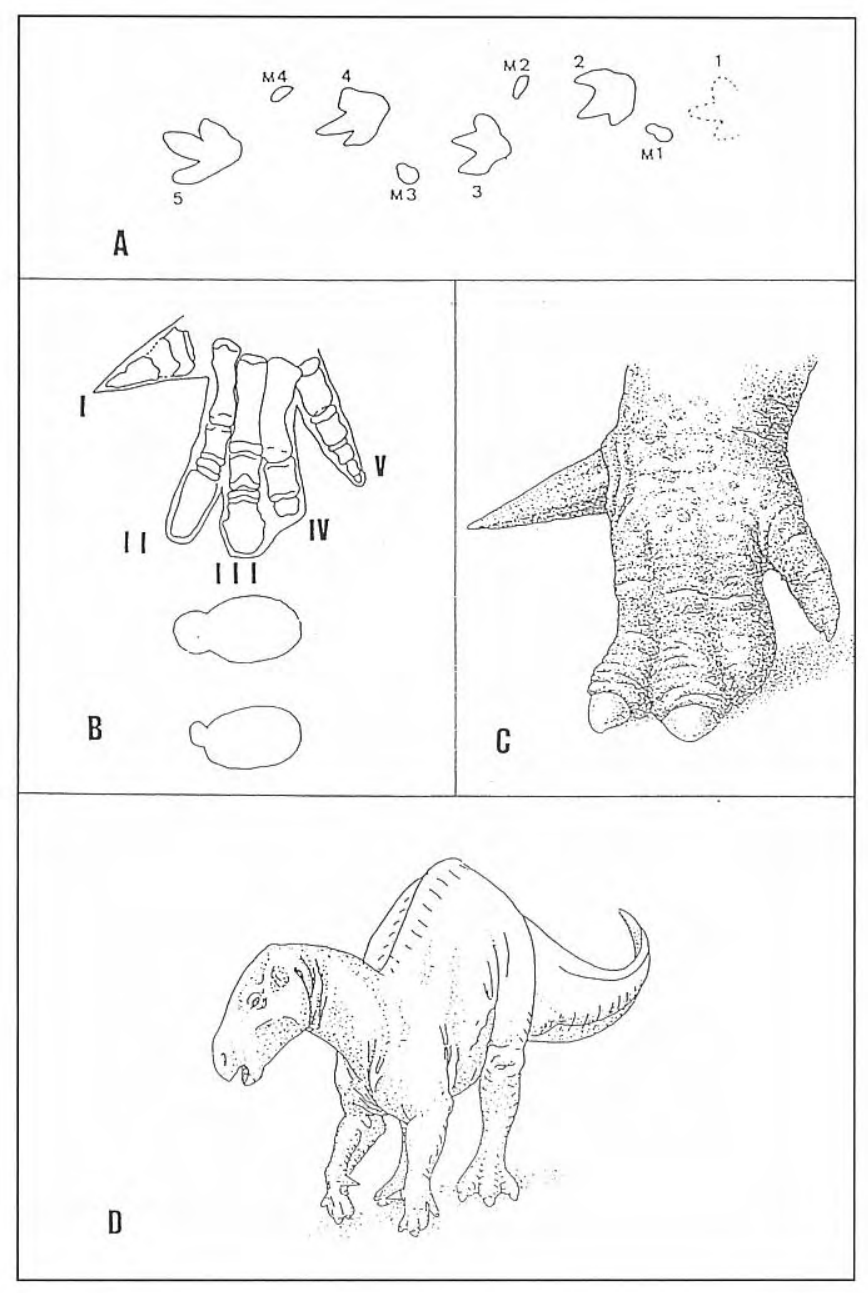

Figura 8. A, Esquema del rastro CBC-R1, del yacimiento Valdemayor (Cabezón de Cameros, La Rioja), producido por un dinosaurio iguanodóntido en progresión cuadrúpeda. B, La morfología bilobulada de las icnitas de las manos permite especular sobre la reconstrucción anatómica del autópodo anterior. Así, el dedo II presentaría una disposición mucho más libre, mientras que los dedos III y IV estarían incluidos en una estructura común de soporte. C, Reconstrucción hipotética de la morfología de la mano. D, Estas consideraciones permiten plantear la reconstrucción en vida de un dinosaurio iguanodóntido en postura cuadrúpeda.

muy grande), lo cual supone un test aceptable para un carácter típico de los dinosaurios: la parasagitalidad de las extremidades. Otra inferencia inherente a los rastros se refiere a la velocidad de progresión del animal responsable. Alexander (1976) estableció de forma empírica una relación entre la velocidad, longitud de zancada y altura de las extremidades en diversos grupos de vertebrados. Aunque este método podría ser considerado más o menos correcto, el problema real, como vimos anteriormente, es la estimación de la altura de la extremidad. En cualquier caso, el método de Alexander ha permitido la consideración del parámetro "longitud de zancada relativa" (razón entre zancada y altura de la extremidad o $\lambda / \mathrm{h}$ ), concepto que proporciona una buena estimación del estado cinético del animal (marcha o carrera) (Alexander, 1976), muy utilizado por los paleoicnólogos. Moratalla (1993) propuso para el mismo el término "índice biodinámico". Demathieu (1984, 1986) propuso también un método alternativo de estimación de la velocidad de marcha, basado en la asimilación del movimiento de la extremidad a un modelo pendular. Aunque es conceptualmente correcto, se trata de un sistema muy difícil en la práctica, ya que necesita una estimación de la dimensión proximal del estilópodo, que en principio no presenta ninguna correlación conocida con las dimensiones de las icnitas.

Los rastros de dinosaurios son muy importantes para entender el patrón de progresión y el modo de apoyo de los autópodos sobre el sustrato. Las pistas de dinosaurios bípedos (tanto terópodos como ornitópodos) muestran en general una rotación interna de sus icnitas (a veces menos evidente en pistas de terópodos) (Fig. 7A y B). Este patrón es sorprendente si consideramos las diferencias morfológicas de las pelvis entre los dos grupos de dinosaurios. La razón de esta rotación icnológica no es del todo evidente, pero probablemente se deba a una ligera rotación pélvica según un eje vertical (Thulborn, 1982) y al movimiento de la cola a derecha-izquierda, como órgano de equilibrio, durante la marcha (Moratalla et al., 1992). Con el fin de guardar un correcto equilibrio, este movimiento podría incrementar la tendencia a orientar ligeramente los pies hacia el interior de la línea media de la pista, es decir, a la utilización de un andar zambo. En los grandes saurópodos, la mayor robustez estructural y la postura cuadrúpeda restarían importancia al papel de la cola como órgano de equilibrio así como a la rotación pélvica. Estas consideraciones se pueden comprobar en el registro fósil. En efecto, las pistas de dinosaurios saurópodos muestran una rotación externa para las icnitas de los pies (Fig. 7C).

La presencia de rastros cuadrúpedos de ornitópodos ha permitido comprobar la hipótesis de su condición cuadrúpeda opcional (Moratalla et al., 1992 y 1994) (Fig. 7B). Los datos de estas pistas cuadrúpedas sugieren que estos dinosaurios utilizaron el mismo grado de rotación interna de los pies, tanto en progresión bípeda como cuadrúpeda. El centro de gravedad se situaría cerca de la cintura pélvica (Alexander, 1989). Esto significa que las manos sólo contactarían suavemente con el sustrato. Como resultado, la utilización de una locomoción cuadrúpeda opcional no modificaría significativamente la rotación de la cintura pélvica o la función de la cola, como órgano de equilibrio, durante la marcha (Moratalla, 1993).

Las icnitas de las manos de estos rastros de ornitópodos permiten proponer hipótesis de reconstrucción anatómica (Fig. 8). La disposición de los metacarpales de los iguanodóntidos, al menos en las formas más graviportales, sugiere que la mano estaba adaptada para soportar peso (Norman, 1980). Los dedos I y V son menores que los centrales y su orientación y tamaño sugieren que no contactaban, durante el apoyo, con el sustrato, excepto quizás en un medio muy fangoso. La unión estructural entre los metacarpales III y IV sugiere, en principio, una 


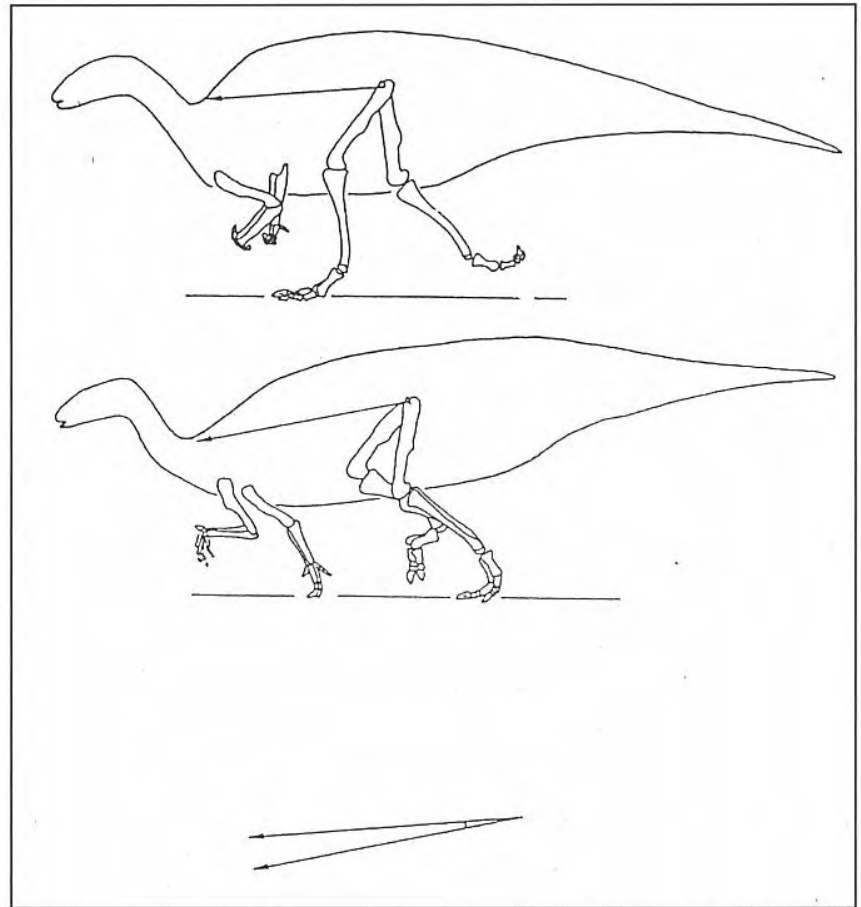

Figura 9. Reconstrucción esquemática de Iguanodon atherfieldensis Hooley (1925) en postura bípeda (redibujado de Norman, 1980). El desplazamiento hacia adelante del centro de gravedad cuando el animal utiliza una progresión cuadrúpeda sería proporcional a la diferencia angular entre las dos posturas. El vector posición del cuerpo ha sido considerado como la línea que une el centro del acetábulo y la apófisis neural de la primera vértebra dorsal. El ángulo estimado en este caso es de unos $7^{\circ}$, sugiriendo que el desplazamiento del centro de gravedad es muy corto. En consecuencia, el cambio a un modo de progresión cuadrúpeda supone un escaso gasto energético (véase también Moratalla et al., 1994).

íntima relación entre ambos dedos, probablemente con disposición paralela y fuertemente yuxtapuestos. Por el contrario, el metacarpal II es más corto, con una disposición no tan unida. El dedo II presenta una gran falange ungueal asimétrica, dirigida medialmente y claramente separada del dígito III, que presenta una falange ungueal más corta y ancha; el dígito IV no tiene falange ungueal. Las icnitas delanteras de los rastros cuadrúpedos de ornitópodos sugieren que la morfología más probable para la mano es una disposición interna y asimétrica del dedo II, con los dedos III y IV probablemente incluidos en una única estructura anatómica (Moratalla et al., 1992). Este patrón podría ser relativamente variable según las especies.

El centro de gravedad de un ornitópodo está situado muy cerca de la cintura pélvica (Alexander, 1989). La postura cuadrúpeda provoca un desplazamiento hacia adelante del centro de gravedad, que será proporcional a la diferencia angular entre las dos posturas (Fig. 9). Las reconstrucciones más fidedignas de estos ornitópodos (i.e.
Norman, 1980) sugieren que esta diferencia angular podría ser muy baja, lo que lleva a interesantes consecuencias: 1) El desplazamiento hacia adelante del centro de gravedad es muy corto. 2) El paso de una postura bípeda a una cuadrúpeda implica un bajo gasto energético. 3) Las extremidades posteriores siempre soportan más peso que las anteriores. 4) Estos factores facilitan la utilización de una postura cuadrúpeda opcional en estos dinosaurios ornitópodos (véase Moratalla et al., 1994).

\section{Asociación de rastros}

Algunos afloramientos paleoicnológicos presentan una entidad suficiente como para permitir la observación de diferentes rastros de dinosaurios impresos en una superficie estratigráfica común. La forma en que se encuentran asociadas estas pistas puede constituir un buen indicio sobre comportamiento en grupo. Es evidente que la presencia de rastros con un sentido de progresión común (lo cual se puede apreciar en muchos yacimientos icnológicos) soporta una interpretación gregarista (Bird, 1944; Ostrom, 1972; Thulborn y Wade, 1984; Leonardi, 1984; Moratalla, 1993). Ésta ha sido siempre la interpretación tradicional de este fenómeno, aunque pueden caber también otras hipótesis, como la probable existencia de condicionantes geográficos (márgenes fluviales, "pasos obligados", etc.). Uno de los aspectos más curiosos del fenómeno de los rastros paralelos suele ser la existencia de un espaciamiento regular entre los mismos, lo que apunta más bien hacia la interpretación gregarista. En ocasiones, existen también solapamientos icnológicos, lo cual pone de manifiesto el orden de paso y permite reconstruir cronológicamente los diferentes eventos de un yacimiento. Muchas veces, los diferentes eventos se han producido incluso en diferentes condiciones del sustrato, con el consiguiente efecto en la morfología de las improntas.

\section{INFORMACIÓN PALEOECOLÓGICA}

Se entiende por Icnocenosis la asociación de rastros o icnitas características de un ambiente particular. Lockley et al. (1994b) proponen que sea referida a un mismo nivel sedimentario. Por el contrario, el término Icnofacies hace referencia a múltiples icnocenosis que tienen una similar composición icnotaxonómica y muestran una asociación recurrente en ambientes particulares definidos. Lockley et al. (1994b) proponen una denominación basada en el icnotaxon más abundante (i.e. Brontopodus icnofacies o Caririchnium icnofacies).

El factor más inmediato en la información paleoecológica inferida a partir del estudio de las icnitas fósiles se refiere al contexto sedimentario en el cual fueron producidas. La formación/conservación de icnitas requiere en general condiciones de baja energía, hecho que puede hacerse extensivo a muy diversos ambientes. Por ejemplo, en zonas litorales restringidas o protegidas de un oleaje importante (lagoons, bahías, etc.), áreas lacustres, llanuras fluviales, etc. En cualquier caso, la formación de huellas necesita de un hiato o interrupción sedimentaria. Es posible 


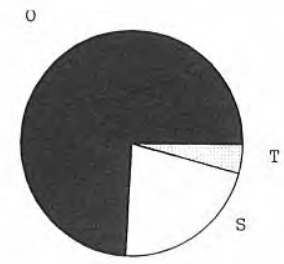

COREA
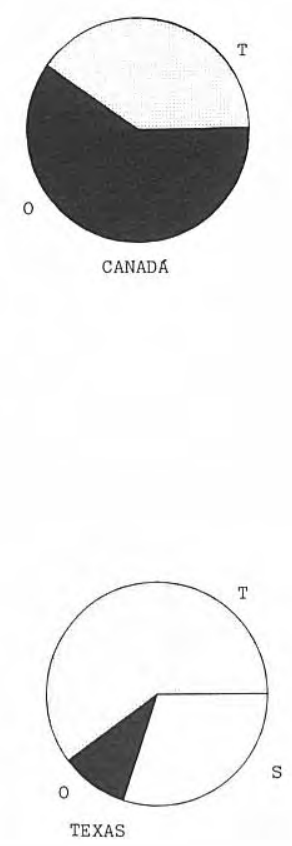

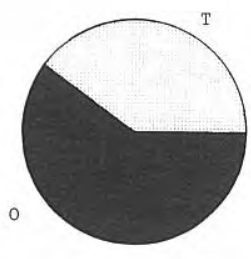

COLORADO
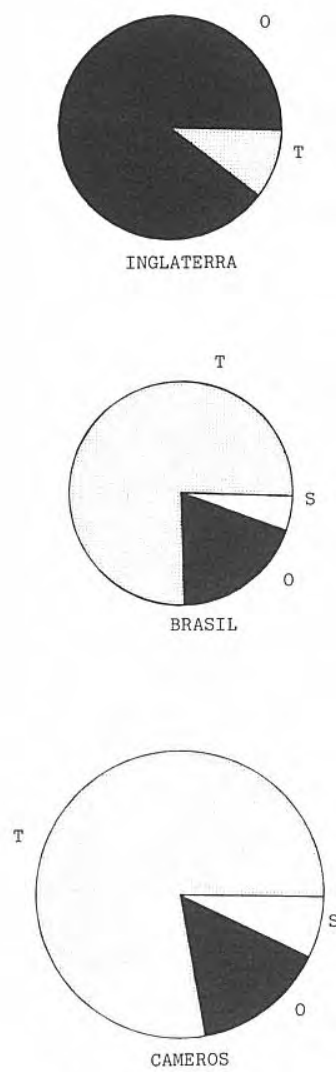

Figura 10. Composición icnofaunística de diversos megayacimientos del Cretácico inferior. T, icnitas de terópodos; $\mathbf{O}$, icnitas de ornitópodos; $\mathrm{S}$, icnitas de saurópodos. Los datos que no hacen referencia a la Cuenca de Cameros están tomados de Lockley y Conrad (1989).

estimar la duración de esta interrupción sedimentaria observando la densidad icnológica, es decir, el índice de dinoturbación (Dodson et al., 1980) (Fig. 10). Sin embargo, hay que tener en cuenta que este índice dependerá del grado de actividad animal, por lo que la tasa de producción de icnitas en un determinado contexto no es constante.

Uno de los aspectos más interesantes de la icnología de dinosaurios es la existencia de regiones muy grandes con diversos o a veces incluso miles de afloramientos de icnitas de dinosaurios, todos incluidos en la misma formación o facies sedimentaria. Estas regiones reciben el nombre de megayacimiento ("megatracksite") (Lockley y Pittman, 1989). Así, un megayacimiento puede ser definido como un estrato o pequeño grupo de estratos icnológicos que cubren grandes áreas geográficas del orden de cientos o incluso miles de kilómetros cuadrados (Lockley, 1991).
Además, otras formaciones geológicas contienen también numerosos yacimientos de icnitas de dinosaurios con relativamente poca extensión lateral, pero un elevado número de niveles estratigráficos. Aunque este tipo de hallazgos no se consideran megayacimientos sensu stricto, Moratalla (1993) propuso para los primeros la denominación megayacimientos de tipo 1 y para este último caso, megayacimientos de tipo 2. Así, los megayacimientos de la Fm. Entrada (Utah) y Dakota Group (Colorado y Nuevo México) son buenos ejemplos del tipo 1. Por el contrario, la Fm. Jindong en Korea (Lim et al., 1989), constituiría un caso del tipo 2. La Cuenca de Cameros constituye un caso peculiar, debido a que se trata de una región muy extensa (alrededor de $8.000 \mathrm{Km}^{2}$ ) con una gran potencia de sedimentos (cerca de 9.000 metros) (Alonso et al., 1991). Una descripción más precisa del megayacimiento de Cameros está siendo desarrollada en un trabajo en preparación.

Lockley (1991) enfatizó la idea de que los megayacimientos han abierto una nueva ventana en la icnología de dinosaurios. En efecto, el estudio de los mismos permite llevar a cabo diversas inferencias, como son censos icnofaunísticos (porcentajes de los diferentes morfotipos icnológicos (Fig. 10), análisis de hábitats, distribución icnológica (en el tiempo y en el espacio), orientaciones preferenciales (significativas para las reconstrucciones paleogeográficas), etc. Debido a la potencialidad de conservación diferencial de restos óseos e icnitas, los primeros no suelen ser abundantes en este tipo de yacimientos. De este modo, las icnitas suelen constituir la gran mayoría de los documentos fósiles de dinosaurios. La Cuenca de Cameros representa, además, un gran intervalo de tiempo, desde el Titónico hasta el Aptiense. Esto permite considerar, hasta cierto punto, eventos evolutivos y reemplazamientos faunísticos. Por supuesto, existen serias limitaciones. En efecto, un censo icnológico presenta dos importantes sesgos: la actividad y el tamaño. Animales más activos serán potencialmente capaces de producir un mayor número de huellas que aquellos animales menos activos. De hecho, diversos megayacimientos (como la Cuenca de Cameros) muestran un gran porcentaje de icnitas de terópodos. Farlow (1987) sugirió que la razón de esta sorprendente composición faunística es la alta tasa de actividad de los carnívoros. Por otro lado, el tamaño constituye también un importante factor de sesgo. Así, la mayoría de huellas de los yacimientos son de media-gran talla, probablemente porque los agentes erosivos pueden destruir o alterar más fácilmente una icnita de menor tamaño.

Como conclusión, se puede afirmar que un buen trabajo en paleoicnología de dinosaurios tratará de poner de manifiesto ambos tipos de información: paleobiológica y paleoecológica. Para la primera, es necesario considerar aquellos factores de sesgo morfológicos, con el fin de identificar, con la mayor precisión posible, las icnitas de dinosaurios. Por consiguiente, se deben aplicar al pasado criterios icnológicos actuales, esto es, el entendimiento de la interacción organismo-sustrato en las formas del presente. Las composiciones icnofaunísticas han de ser analizadas a la luz de los factores conservacionales de este 
tipo de restos fósiles. En caso contrario, se pueden proponer conclusiones no plenamente justificadas.

\section{AGRADECIMIENTOS}

Este trabajo forma parte del proyecto financiado por IBERDROLA para el estudio de los yacimientos de icnitas de dinosaurios del Mesozoico español. Agradecemos sinceramente a los Dres. José Carlos García Ramos y M ${ }^{a}$ Lourdes Casanovas, por la revisión crítica del manuscrito y proponer valiosas sugerencias que han permitido ultimar el texto, tanto en su forma como en sus contenidos.

\section{BIBLIOGRAFÍA}

Abel, O. 1935. Vorzeitliche Lebensspuren. Gustav Fischer, Jena, $644 \mathrm{pp}$.

Alexander, R. McN. 1976. Estimates of speeds of dinosaurs. Nature, 261, 129-130.

Alexander, R. McN. 1989. Dynamics of dinosaurs and other extinct giants. Columbia University Press, New York, 167 pp.

Alonso, A., Meléndez, N. y Mas, J.R. 1991. Sedimentación lacustre durante el Cretácico en la Cordillera Ibérica, España. Acta Geológica Hispánica, 26(1), 35-54.

Baird, D. 1957. Triassic reptile footprints faunules from Milford, New Jersey. Bulletin of the Museum of Comparative Zoology at Harvard College, 117, 449-520.

Bird, R.T. 1944. Did Brontosaurus ever walk on land? Natural History, 53, 61-67.

Bromley, R.G. 1990. Trace Fossils. Biology and Taphonomy. Unwin Hyman, London, 280 pp.

Cohen, A., Lockley, M.G., Halfpenny, J. and Ellinor Michel, A. 1991. Modern Vertebrate Track Taphonomy at Lake Manyara, Tanzania. Palaios, 6, 371-389.

Demathieu, G.R. 1984. Utilisation de lois de la mécanique pour l'estimation de la vitesse de locomotion des Vértebrés tétrapodes du passé. Geobios, 17, 439-446.

Demathieu, G.R. 1986. Nouvelles recherches sur la vitesse des Vértebrés auteurs de traces fossiles. Geobios, 19, 327-333.

Demathieu, G.R. and Wright, R.V.S. 1988. A new approach to the discrimination of chirotherioid ichnospecies by means of multivariate statistics. Triassic eastern border of the French Massif Central. Geobios, 21, 729-739.

Dodson, P., Behrensmeyer, A.K., Bakker, R.T. and McIntosh, J.S. 1980. Taphonomy and paleoecology of the dinosaur beds of the Jurassic Morrison Formation. Paleobiology, 6, 208-232.

Dollo, L. 1883. Troisème note sur les dinosuriens de Bernissart. Bulletin di Musée Royal, 2 85-120.

Farlow, J.O. 1987. A Guide to Lower Cretaceous Dinosaur Footprints and Tracksites of the Paluxy River Valley, Somervell County, Texas. Field trip guidebook, SouthCentral Section, Geological Society of America (Waco, Texas: Baylor University), $50 \mathrm{pp}$.

Farlow, J.O. 1993. The Dinosaurs of Dinosaur Valley State Park (Ed. G. Zappler). Texas Parks and Wildlife Department, Austin, Texas, 32 pp.
Farlow, J.O., Pittman, J.G. and Hawthorne, J.M. 1989. Brontopodus birdi, Lower Cretaceous Sauropod Footprints from the U.S. Gulf Coastal Plain. In: Dinosaur Tracks and Traces (Eds. D.D. Gillette \& M.G. Lockley). Cambridge University Press, Cambridge, 371-394.

Haubold, H. 1984. Saurierfährten. Die Neue Brehm-Bücherei 479, A. Ziemse Verlag, Wittenberg Lutherstadt, Germany, $231 \mathrm{pp}$.

Hersberger, J. and Farlow, J.O. 1990. Tracking dinosaurs with trigonometry. Applications, 83(1), 46-50.

Hitchcock, E. 1858. Ichnology of New England. A report of the sandstone of the Connecticut Valley especially its footprints. W. White, Boston, 220 pp.

Hooley, R.W. 1925. On the skeleton of Iguanodon atherfieldensis sp. nov., from the Wealden shales of Atherfield (Isle of Wight). Quarterly Journal of the Geological Society of London, 81, 1-61.

Ishigaki, S. 1986. Dinosaur footprints of the Atlas Mountains. Nature Study, 32(1), 6-9.

Ishigaki, S. 1989. Footprints of Swimming Sauropods from Morocco. In: Dinosaur Tracks and Traces (Eds. D.D. Gillette \& M.G. Lockley). Cambridge University Press, Cambridge, 83-86.

Langston, W. Jr. 1979. Lower Cretaceous Dinosaur Tracks near Glen Rose, Texas. In: Lower Cretaceous shallow marine environments in the Glen Rose Formation: Dinosaur Tracks and Plants. Field Trip Guide. American Association of Stratigraphic Palynologists. 12th Annual Meeting, Dallas, Texas, 39-61.

Leonardi, G. 1984. Le impronte fossili di Dinosauri. In: Sulle Orme dei Dinosauri (Eds. J.F. Bonaparte, E.H. Colbert, P.J. Currie, A. de Ricqlès, Z. Kielan-Jaworowska, G. Leonardi, N. Morello and P. Taquet), 165-168.

Leonardi, G. (Ed.) 1987. Glosary and Manual of Tetrapod Footprint Palaeichnology. Ministerio das Minas e Energia. Departamento Nacional de Produçao Mineral, Brasilia. 75 pp.

Lim, S.K., Yang, S.Y. and Lockley, M.G. 1989. Large Dinosaur Footprint Assemblages from the Cretaceous Jindong Formation of Southern Korea. In: Dinosaur Tracks and Traces (Eds. D.D. Gillette \& M.G. Lockley). Cambridge University Press, Cambridge, 333-336.

Lockley, M.G. 1986. The Paleobiological and Paleoenvironmental Importance of Dinosaur Footprints. Palaios, 1, 3747.

Lockley, M.G. 1991. Tracking Dinosaurs. Cambridge University Press, Cambridge. 250 pp.

Lockley, M.G. 1994. Dinosaur ontogeny and population structure: Interpretations and speculations based on fossil footprints. In: Dinosaur Eggs and Babies (Eds. K. Carpenter, K.F. Hirsch \& J.R. Horner). Cambridge University Press, Cambridge, 347-365.

Lockley, M.G. and Conrad, K. 1989. The Paleoenvironmental Context, Preservation and Paleoecological Significance of Dinosaur Tracksites in the Western USA. In: Dinosaur Tracks and Traces (Eds. D.D. Gillette \& M.G. Lockley). Cambridge University Press, Cambridge, 121-134.

Lockley, M.G. and Hunt, A.P. 1995. Dinosaur tracks and other fossil footprints of the western United States. Columbia University Press, New York, 338 pp. 
Lockley, M.G. and Pittman, G.J. 1989. The Megatracksite Phenomenon: Implications for Paleoecology, Evolution and Stratigraphy. Journal of Vertebrate Paleontology, 9, 30A.

Lockley, M.G. and Rice, A. 1990. Did "Brontosaurus" ever swim out to sea?: evidence from brontosaur and other dinosaur footprints. Ichnos, 1, 81-90.

Lockley, M.G., Hunt, A.P. and Meyer, C.A. 1994a. Vertebrate tracks and the ichnofacies concept: implications for Palaeoecology and Palichnostratigraphy. In: The Palaeobiology of Trace Fossils (Ed. S.K. Donovan). John Wiley \& Sons, Chichester, 241-268.

Lockley, M.G., Hunt, A.P. and Moratalla, J.J. 1994b. Limping dinosaurs? Trackway evidence for abnormal gaits. Ichnos, 3, 1-10.

Moratalla, J.J. 1993. Restos indirectos de dinosaurios del registro español: Paleoicnología de la Cuenca de Cameros (Jurásico superior-Cretácico inferior) y Paleoología del Cretácico superior. Tesis Doctoral. Universidad Autónoma de Madrid. Dpto. Biología. 727 pp.

Moratalla, J.J., Sanz, J.L. and Jiménez, S. 1988. Multivariate analysis on Lower Cretaceous dinosaur footprints: discrimination between ornithopods and theropods. Geobios, 21, 395-408.

Moratalla, J.J., Sanz, J.L., Jiménez, S. and Lockley, M.G. 1992. A quadrupedal ornithopod trackway from the Lower Cretaceous of La Rioja (Spain): inferences on gait and hand structure. Journal of Vertebrate Paleontology, 12(2), 150-157.

Moratalla, J.J., Sanz, J.L. and Jiménez, S. 1994. Dinosaur tracks from the Lower Cretaceous of Regumiel de la Sierra (province of Burgos, Spain): inferences on a new quadrupedal ornithopod trackway. Ichnos, 3, 89-97.

Norman, D.B. 1980. On the ornithischian dinosaur Iguanodon bernissartensis from the Lower Cretaceous of Bernissart (Belgium). Institut Royal des Sciences Naturelles de
Belgique, 178, 1-103.

Ostrom, J.H. 1972. Were some dinosaurs gregarious? Palaeogeography, Palaeoclimatology, Palaeoecology, 11(4), 287-301.

Peabody, F.E. 1955. Taxonomy and the footprints of Tetrapods. Journal of Paleontology, 29, 915-918.

Sanz, J.L., Moratalla, J.J. y Casanovas, M.L. 1985. Traza icnológica de un Dinosaurio Iguanodóntido en el Cretácico inferior de Cornago (La Rioja, España). Estudios geológicos, 41, 85-91.

Sarjeant, W.A.S. 1975. Fossil tracks and impressions of vertebrates. In: The study of Trace Fossils (Ed. R.W. Frey). Springer Verlag, Berlin, 283-324.

Sarjeant, W.A.S. 1989. 'Ten Paleoichnological Commandments': A Standarized Procedure for the Description of Fossil Vertebrate Footprints. In: Dinosaur Tracks and Traces (Eds. D.D. Gillette \& M.G. Lockley). Cambridge University Press, Cambridge, 369-370.

Sarjeant, W.A.S. and Leonardi, G. 1987. Substrate and Footprints. In: Glosary and Manual of Tetrapod Footprint Palaeoichnology (Ed. G. Leonardi). Ministerio das Minas e Energia. Departamento Nacional da Produçao Mineral, Brasilia, 53.

Thulborn, R.A. 1982. Speeds and gaits of Dinosaurs. Palaeogeography, Palaeoclimatology, Palaeoecology, 38, 227-256.

Thulborn, R.A. 1990. Dinosaur Tracks. Chapman \& Hall, London. 410 pp.

Thulborn, R.A. and Wade, M. 1984. Dinosaur trackways in the Winton Formation (mid-Cretaceous) of Queensland. Memoirs of the Queensland Museum, 21, 413-517.

Weems, R.E. 1992. A re-evaluation of the taxonomy of Newark Supergroup Saurischian dinosaur tracks, using extensive statistical data from a recently exposed tracksite near Culpeper, Virginia. Proceeding 26th Forum on the Geology of Industrial Minerals, May 1990, 113-127. 